1973-06-01

\title{
An intensity-dependent filter
}

James M. Thorne

Thomas R. Loree

George McCall

Follow this and additional works at: https://scholarsarchive.byu.edu/facpub

Part of the Electrical and Computer Engineering Commons

\section{Original Publication Citation}

Thorne, J., T. Loree, and G. McCall. "An Intensity-Dependent Filter." Quantum Electronics, IEEE Journal of 9.6 (1973): 637-8

\section{BYU ScholarsArchive Citation}

Thorne, James M.; Loree, Thomas R.; and McCall, George, "An intensity-dependent filter" (1973). Faculty Publications. 786.

https://scholarsarchive.byu.edu/facpub/786

This Peer-Reviewed Article is brought to you for free and open access by BYU ScholarsArchive. It has been accepted for inclusion in Faculty Publications by an authorized administrator of BYU ScholarsArchive. For more information, please contact ellen_amatangelo@byu.edu. 
esting comparison in that they have similar masses and static electric dipole moments but differ substantially in magnetic environment due to the unpaired $\pi$-orbital of the latter. In selected cases the molecular angular momentum $(j)$ dependence is also examined.

2.8 Detailed Behavior of Gaseous Nonlinear Absorbers as Nonlinear Elements for Laser Systems, C. P. Christensen and S. E. Schwarz, Department of Electrical Engineering and Computer Sciences, University of California, Berkeley, Calif. 94720 .

$(15 \mathrm{~min})$

Saturable-absorber gases are used with infrared lasers, such as the $\mathrm{CO}_{2}$ laser, for $Q$ switching, mode locking, and other purposes. Earlier work, notably that of Stein feld et al., ${ }^{1}$ has discussed the probable internal mechanisms of such absorbers and given values of some of the relevant parameters. In most cases an absorber such as $\mathrm{SF}_{\theta}$ has been modeled as a four-level system, in which the lower absorbing level is coupled by a fast relaxation to a lower "bath" of levels and the upper level of the absorbing transition is similarly coupled to an upper bath. The behavior of the absorber is then described by means of rate equations, the parameters of which are relaxation rates and also the numbers of levels in the baths.

We have performed an experimental study of gaseous $\mathrm{SF}_{\mathrm{e}}$ under conditions similar to those occurring in mode-locked lasers. A stable $\mathrm{CW} \mathrm{CO}_{2}$ laser is amplitude modulated and the modulated beam is then passed through the nonlinear absorber. A second unmodulated beam with perpendicular polarization is used to measure by absorption the time-varying populations of energy levels in the gas. The modulation frequency of the exciting beam can be varied between zero and $10 \mathrm{MHz}$, so that amplitudes of the variations in level populations can be studied as a function of frequency. The phases of these variations have also been measured as functions of frequency at the lower frequencies. This type of experiment is more sensitive to details of the nonlinear absorption than the pulsed technique of Steinfeld et al. Essentially, it gives a direct measurement of the nonlinear coefficient responsible for mode locking. Moreover it provides measurements of the parameters of the four-level model and gives some interesting physical insight into the behavior of the nonlinear absorber.

The case in which the probe beam has the same frequency as the pumping beam (before the latter is modulated) resembles the situation most often arising in modelocked lasers, with the modulation frequency playing the role of the intermode frequency spacing. (Our modulation fre-

1 J. I. SteinfeId, I. Burak, D. G. Sutton, and A. V. Nowak, "Infrared double resonance in sulfur hexafluoride," J. Chem. Phys., vol. 52 pp. 5421-5434, May 1970. quencies are somewhat lower than typical intermode frequencies, but relaxation rates and thus frequencies can be "scaled" by changing the gas pressure.) We find that at frequencies above an initial low-frequency rolloff due to thermal diffusion, the nonlinear coefficient remains nearly constant until a value essentially equal to the kinetictheory collision frequency is reached, after which it rolls off at approximately $20 \mathrm{~dB}$ / decade. The constancy of the nonlinear coefficient in the mid-frequency region is somewhat remarkable, since one might expect that the existence of distinct relaxation rates between some levels within the baths would give rise to detailed structure in the curve of nonlinear coefficient versus frequency. (Indeed, even the relaxation rate between the two baths - the $V-T$ ratefails to give rise to structure at the corresponding frequency.) These results are in very good agreement with the four-level model. The agreement is surprisingly good, considering the simplicity of the model.

Another interesting finding results when the probing beam has a frequency more than $50 \mathrm{GHz}$ removed from that of the pumping beam. This case is relevant to a possible "line-locked" $\mathrm{CO}_{2}$ laser, in which modes corresponding to different rotational lines of $\mathrm{CO}_{2}$ would be locked in phase so as to give ultrashort pulses. We find, however, that the nonlinear coupling between waves spaced farther apart in frequency than the kinetic collision frequency tends to decrease rapidly with increasing frequency spacing. This indicates that line locking will be favored by the use of highpressure absorbers and also by the use of long cavities. The latter will allow closely spaced adjacent modes to interact strongly, even though more widely spaced pairs of modes may not interact to any great extent.

Our measurements of the principal relaxation rates of $\mathrm{SF}_{8}$ are in good agreement with those found in the literature. However, the effective number of levels in the lower bath (the more important of the two) is found to be at least ten times larger than has been reported in earlier work. This in turn implies that the transition dipole moments are larger than previously reported.

2.9 An Intensity-Dependent Filter, ${ }^{1}$ James M. Thorne, Chemistry Department, Brigham Young University, Provo, Utah 84601, Thomas R. Loree, and Gene H. McCall, University of California, Los Alamos Scientific Laboratory, Los Alamos, N. Mex. 87544.

$(15 \mathrm{~min})$

It is useful in optically driven Kerr-cell shutters and other laser applications to have intense pulses with extremely low light levels between pulses. We describe here a device that accomplishes this objective external to the laser cavity.

${ }^{1}$ This work was supported by the $\mathbf{U}$. S. Atomic Energy Commission.
In our system, we use a Nd-YAG laser oscillator, mode locked with Eastman 9740 dye in dichloroethane. The energy of the eight-pulse train is approximately $8 \mathrm{~mJ}$. Individual pulses are approximately $20 \mathrm{ps}$ wide.

The optical train of the filter consists of the following:

1) a polarizer oriented at $0^{\circ}$ to pass the maximum amount of laser light;

2 ) an $\frac{1}{8}$ wave plate with fast axis oriented at $+45^{\circ}$ to elliptically polarize the light;

3) a 1-cm cell of carbon disulfide $\left(\mathrm{CS}_{2}\right)$ which rotates the major axis of the elliptically polarized light in direct proportion to its intensity ${ }^{2}$;

4) an $\frac{1}{8}$ wave plate with fast axis oriented at $-45^{\circ}$ to convert the light back to linear polarization;

5) a $1-\mathrm{cm}$ cell of Eastman 9740 modelocking dye (absorbance $=1.7$ ) placed at the point where the most intense pulses are focused by the mild self-focusing in the $\mathrm{CS}_{2}$. This focusing enhances the normal dye bleaching by intense light. Recovery time is approximately 10 ps. Lower intensity light is not focused to any appreciable extent and is mostly absorbed by the dye;

6) a polarizer oriented at $90^{\circ}$ to block light that has not been rotated (low intensity light is rotated very little by the $\mathrm{CS}_{2}$ );

7) a lens system to recollimate the transmitted light.

It can be seen that two independent mechanisms are involved in the discrimination against low-intensity light and that the $\mathrm{CS}_{2}$ is central to both. First, the $\mathrm{CS}_{2}$ focuses intense pulses on the dye cell and enhances bleaching and transmission. Second, the $\mathrm{CS}_{2}$ rotates the plane of polarization of intense pulses so they pass through the second polarizer more readily. This second effect is described by ${ }^{3}$ :

$$
I_{\text {out }}=K\left(I_{\text {in }}\right)^{3}
$$

when the rotation is small enough for the cosine of the rotation $\beta$ to be approximated by $1-\beta$. In (1), $I_{\text {in }}$ is the intensity incident on the $\mathrm{CS}_{2}$ cell, $I_{\text {out }}$ is the intensity transmitted by the system with no dye cell, and $K$ is a constant that depends on the retardation and orientation of the retardation plates, and on the length and nonlinear susceptibility of the $\mathrm{CS}_{2}$.

The transmission of the $\mathrm{CS}_{2}-$ dye combination (polarizers not considered) can be obtained from ${ }^{4}$ :

$$
P / P_{c}=\{[\ln D T / F(T-1)]+G\}^{-1}
$$

where $P$ is the laser power, $P_{c}$ the critical power for the $C_{3}$, and $D, F$, and $G$ are parameters that depend on the low light

${ }^{2}$ P. D. Maker, R. W. Terhune, and C. M. Sayage, Phys, Rev, Lett. vol. 12, p. 507, 1964. ${ }^{3}$ J. M. Thorne, T. R. Loree, and G. H. McCall, Appl. Phys. Lett., to be published.

${ }^{4}$ J. M. Thorne and T. R. Loree, Opt. Commun., to be published. 
level transmission of the dye and the geometry of the system.

The transmission of the combined system is extremely sensitive to $\mathrm{CS}_{2}$ path length, dye concentration, component spacing, and incident light intensity. Data will be presented to show how combinations of these parameters can be varied to achieve the desired filter characteristics with regard to cutoff intensity and steepness of cutoff.

2.10 A Laser Oscillator that Directly Produces Bandwidth-Limited Pulses in the 1-ns Region and an Amplifier Chain that Maintains the Single-Pulse Coherence, ${ }^{1}$ B. C. Johnson, L. L. Steinmetz, R. L. Carman, and W. H. Lowdermilk, Lawrence Livermore Laboratory, University of California, Livermore, Calif. 94550 .

$(15 \min )$

We report on a laser oscillator that directly produces bandwidth-limited output pulses (i.e., pulses lacking frequency or amplitude modulation) with durations ranging from $<0.5$ to $>2.5 \mathrm{~ns}$.

The oscillator operates as a driven optical resonator. Intracavity spontaneous emission noise is gated symmetrically in time by a fast-switching Pockels cell through a high- $Q$ state and back to a low- $Q$ state This initial noise pulse is then allowed to build up under the constraint of controlledbandwidth feedback. ${ }^{2}$ By appropriately matching the temporal properties of the intracavity $Q$-switch gate (which switches rapidly compared to the cavity round trip time) to the allowable frequency line width (determined by the optical elements in the cavity and the system gain), we obtain bandwidth-limited outputs. In principle, any pulse duration less than the cavity round-trip time can be achieved by the techniques we describe here. We are currently operating a Nd:YAG system, but the principle is also applicable to other active media.

The optical cavity of the oscillator is defined by two multiple-faced resonantly reflecting etalons. Within this cavity are the following elements: 1) a gain medium (e.g., Nd:YAG), 2) a polarizing element, 3) a quarter-wave (QW) plate, and 4) a fast electrooptic $Q$-switching cell. There are two modes of operation for the system. In the first, or self-driven mode, the QW plate is oriented for near maximum retardation and the Pockels cell (PC) is biased initially at its half-wave retardation value. ${ }^{2}$ In the second, or synchronously driven mode, the QW plate is set for zero retardation (or else removed from the cavity) and the $\mathrm{PC}$ is biased at its quarter-

${ }^{1}$ This work was supported by the U. S. Atomic Energy Commission.

${ }^{2}$ R. L. Carman, L. L. Steinmetz, and B. C. Johnson, 6th All Soviet Union Conf. Nonlinear Optics, Minsk, USSR, July 1972. wave value. In both cases, there is approximately zero transmission through the cavity initially. At maximum inversion, the cavity is switched by shorting the PC bias voltage either 1) through the QW value to ground (self-driven mode) or 2) through zero to its negative QW value (synchronously driven mode). In each instance, the effect is to introduce into the cavity a spontaneous emission noise pulse with a $\sin ^{2}(t)$ time dependence and amplitude half-width determined by the time it takes to switch the PC. The initial pulse has the following properties: 1) spatial extent less than the cavity length; 2) envelope time symmetric about its intensity maximum; and 3 ) initial bandwidth of up to the full fluorescent line width of the gain medium. Once initiated, this pulse then proceeds to build up toward a gain-saturating pulse. On each pass, the narrow-band feedback of the resonant reflectors acts to limit the pulse bandwidth, with an effective Fabry-Perot finesse factor equal to the number of round trips times the finesse per pass. In the self-driven mode, pulse buildup occurs with the PC near zero retardation and the cavity in a low $Q$ or lossy condition. In the synchronously driven mode, we permit the voltage applied to the PC to "ring" alternately between $+V_{Q w}$ and $-V_{Q w}$ and adjust the zero crossing (maximum cavity transmission) points to coincide in time with the round-trip time for the initially gated pulse.

The oscillator operates satisfactorily in both modes, with an energy/pulse $\sim 10$ times greater for the synchronously driven system, due to the lower effective loss presented to the oscillating pulse. In either case, it is possible to control the pulse duration and spectral content up to the uncertainty principle limit. The independent determination of these two parameters makes it possible to obtain bandwidthlimited output pulses from the oscillator over a range of pulse durations.

We present experimental results on the Nd:YAG oscillator and amplifier system. The switching time of the oscillator PC is adjusted to produce 1-ns pulses. The total frequency selection provided by the cavity is then calculated from known properties of the resonant reflectors and the system gain, and is found to agree closely with the inverse of the 1-ns pulse duration. Streaking camera (10-ps resolution) and 519 oscilloscope (300-ps risetime) results show the pulses to be Gaussian in time, with no amplitude modulation. Frequency measurements taken with a spectrograph $\left(<0.1 \mathrm{~cm}^{-1}\right.$ resolution) and Fabry-Perot etalon $\left(\sim 0.01 \mathrm{~cm}^{-1}\right.$ resolution) show the pulses consist of a single spectral line with a frequency width of $\sim 0.03 \mathrm{~cm}^{-1}$.

A single $(0.5-\mathrm{mJ}$ 1-ns 1 -mm-diam) pulse from the oscillator has been amplified to $\sim 1 \mathrm{~J}$ in a system consisting of a spatially filtered telescope, two Nd:YAG $(1 \mathrm{~cm}$ diam by $7.5 \mathrm{~cm}$ long) and two Nd:glass (1.4 $\mathrm{cm}$ diam by $22.5 \mathrm{~cm}$ long) amplifiers. The high quality of the initial pulse (i.e., Airy central disk spatial and smooth Gaussian temporal variation) was maintained throughout the system. Further data will be presented dealing with pulse-distortion effects for energy outputs $>1 \mathbf{J}$ due to amplifier saturation.

\section{Session 3}

Wednesday, May 30, 1973

9:00-11:45 A.M.

\section{Optical-Fiber Transmission}

Chairman: Tingye Li, Bell Laboratories, Holmdel, N.J.

3.1 Optical Fibers for Communication (Invited), D. Gloge, Bell Telephone Laboratories, Inc., Crawford Hill Laboratory, Holmdel, N. J. 07733.

(30 $\mathrm{min})$

Glass fibers are as thin and flexible as a human hair and easily produced in lengths of miles. Their transparency in the visible and near infrared-improved beyond all expectations by recent breakthroughs ${ }^{1}$ seems now sufficient to transmit optical signals unprocessed over miles. No wonder then, that efforts have intensified all over the world to utilize fibers in future communication systems. This paper attempts to highlight some of the diverse approaches being followed in pursuit of the common goal.

Materials research and fabrication are probably the fields where present progress is most rapid. The ideas and possibilities are manyfold. Traditionally, the way of forming a low-index cladding around a high-index fiber core is by pulling the fiber from two concentric crucibles or from a rod-and-tube preform. New ways of preform preparation by deposition, doping, or diffusion are being studied and may offer improvements and versatility. As far as materials are concerned, fused silica have shown the lowest bulk losses and hence receive the most interest, but many glasses are being studied as well. The search even goes beyond the glassy materials: certain pure liquids were found to form excellent waveguides when filled into capillary glass tubes.

As new processes become available and record "lows" in fiber loss are being reached, propagation theory is finding new challenges as well. What influence, for example, have bends or diameter variations along the fiber length and what tolerances can be allowed? Or how can delay distortion be controlled? On the one hand, multimode fibers seem desirable with respect to transmitter compatibility, splicing, and fabrication tolerances. On the other hand the signal distortion caused by mode delay differences in multimode fibers can be considerable and may require some equalization-inherent in the fiber or at the fiber end. Some concepts are discussed in this talk. Yet even complete equalization of these delay differences may leave dispersion effects serious enough to be of importance.

Thinking ahead, one is confronted with the question of fiber handling, strength, and life. The technology of making cables and

1 For example, Corning's multimode fiber having a loss of $4 \mathrm{~dB} / \mathrm{km}$ (Corning Glass Works, Aug. 14, 1972). 